\title{
A neurophysiological approach to nerve transfer to restore upper limb function in cervical spinal cord injury
}

\author{
Ross M. Mandeville, MD, ${ }^{1}$ Justin M. Brown, MD, ${ }^{2}$ and Geoffrey L. Sheean, MD1 \\ Departments of ${ }^{1}$ Neurosciences and ${ }^{2}$ Neurosurgery, University of California San Diego School of Medicine, La Jolla, California
}

A successful nerve transfer surgery can provide a wealth of benefits to a patient with cervical spinal cord injury. The process of surgical decision making ideally uses all pertinent information to produce the best functional outcome. Reliance on clinical examination and imaging studies alone can miss valuable information on the state of spinal cord health. In this regard, neurophysiological evaluation has the potential to effectively gauge the neurological status of even select pools of anterior horn cells and their axons to small nerve branches in question to determine the potential efficacy of their use in a transfer. If available preoperatively, knowledge gained from such an evaluation could significantly alter the reconstructive surgical plan and avoid poor results. The authors describe their institution's approach to the assessment of patients with cervical spinal cord injury who are being considered for nerve transfer surgery in both the acute and chronic setting and broadly review the neurophysiological techniques used.

https://thejns.org/doi/abs/10.3171/2017.5.FOCUS17245

KEY WORDS spinal cord injuries; neurophysiology; nerve transfer; electromyography; quadriplegia; reconstructive neurosurgery

$\mathrm{P}$ ATIENTS with cervical spinal cord injury (SCI) have residual neurological function above the level of their lesion and reduced or no function at and below the lesion level. ${ }^{32}$ With midcervical cord injuries, muscles in the shoulder girdle and proximal upper limb, and sometimes in the forearm, may retain good voluntary control and force generation. ${ }^{7}$ Partial nerve transfers from these muscles to muscles below the lesion level have been used with success by reconstructive neurosurgeons to restore function. 7,12,16,23,55 Intuitively, if a muscle appears to have good bulk and strength, a transfer from the nerve innervating that muscle should have a good chance of success, all else being equal. In practice, however, this is not always the case. ${ }^{36}$ Sometimes muscles innervated by the donor nerve are rendered too weak by the transfer and remain so. Sometimes the recipient muscle does not develop the desired level of innervation and strength. Understanding the pathophysiology of SCI and the physiology of nerve transfer helps to explain why poor results are encountered and reveals methods to improve success.

\section{Pathophysiology of SCI}

Spinal cord injuries are either complete or incomplete. ${ }^{14,32}$ Acutely, there is a reduction of strength and sensation at and below the lesion. Deficits are usually due to a combination of spinal tract and anterior horn cell (AHC) lesions within the cord, but there tends to be preferential destruction of the central gray matter containing those AHCs at the level of injury. The lower motor neuron (LMN) lesions that result from the loss of AHCs, as well as trauma to adjacent nerve roots, affect muscle segments at, above, or below the injured metamere. As a result of this gray matter destruction, which often extends both rostral

ABBREVIATIONS AHC = anterior horn cell; CMAP = compound muscle action potential; EMG = electromyography; IP = interference pattern; IQ = innervation quotient; $\mathrm{LMN}=$ lower motor neuron; MRC = Medical Research Council; MU = motor unit; MUNE = MU number estimation; MUNIX = MU number index; MUP = MU potential; SCI = spinal cord injury; UMN = upper motor neuron.

SUBMITTED April 26, 2017. ACCEPTED May 5, 2017.

INCLUDE WHEN CITING DOI: 10.3171/2017.5.FOCUS17245. 
and caudal to the dominant site of injury, potential donor and recipient nerves can be denervated to some degree. This has implications for nerve transfers. ${ }^{7,8,10}$

Delayed effects of SCI are well known..$^{15}$ Features of the upper motor neuron (UMN) syndrome include muscle overactivity, including spasticity, spastic co-contraction, and flexor-extensor spasms. In addition, over time soft tissue changes can further contribute to resistance to passive movement and reduced range of motion if aggressive therapy is not pursued. Unlike brain lesions, in motor complete spinal cord lesions, there is little potential to compensate for the loss of UMN control. ${ }^{15,56}$

\section{Collateral Reinnervation After LMN Lesions}

After partial LMN lesions, remaining axons sprout from their preterminal portions to innervate nearby denervated muscle fibers, thereby creating a larger motor unit (MU). ${ }^{1}$ Such collateral reinnervation can be remarkably successful in restoring strength and bulk.,13,27,28 This principle, exploited by nerve transfer techniques, allows partially denervated donor muscles (from which axons have been "borrowed") the opportunity to recover significantly, avoiding an associated deficit. However, this compensatory mechanism has its limits. ${ }^{54}$ Beyond a threshold fraction of damaged axons, the mechanism fails to restore full strength ${ }^{44}$ Furthermore, the characteristics of remaining MUs are important. The spatial volume of muscle that an MU is capable of innervating tends to be near constant in adults: ${ }^{19,21,31}$ that is, there is a physical spatial limit beyond which sprouting is not likely to expand. Thus, the distribution of remaining MUs needs to broadly cover the muscle to have any hope of reinnervating all muscle fibers; denervated muscle fibers lying outside all remaining MUs' potential territories will not benefit from collateral reinnervation. A second limitation to collateral reinnervation lies in the fact that the absolute number of muscle fibers that each remaining MU can support is limited.$^{60}$ Therefore, even if a muscle fiber lies within an MU's territory, it may not be reinnervated via collateral sprouting if that MU has reached its innervation capacity. Postpolio patients who suffer denervation at a young age exemplify well the size that MU potentials (MUPs) can become electrophysiologically. ${ }^{20,48}$ However, in these cases, the impressively large MUP is thought to be secondary to not just the great number of muscle fibers innervated by that individual MU, but also a significant degree of muscle fiber hypertrophy. ${ }^{50}$ This hypertrophy causes the recorded MUP to be even larger than expected for the number of muscle fibers in its territory and presumably provides greater force overall per MU. Unfortunately, an MU's ability to support such a cohort of muscle fibers and the muscle fiber's ability to undergo such hypertrophy appear to decline with age and the level of collateral reinnervation, and force per MU can be expected to fall accordingly. ${ }^{17,30}$

The implication of collateral reinnervation, as described above, is that potential donor muscles may have good bulk and strength achieved by relatively few large MUs. Thus, while this muscle may generate a reasonable amount of strength, a subsequent transfer of fibers from its innervating nerve may not only result in the severe loss of donor strength, but also provide poor innervation to the recipient. This problem is further compounded because the number of functioning axons in the transfer is again pruned as they traverse the surgical coaptation site. ${ }^{11}$ We have found that a Medical Research Council (MRC) Grade 4 muscle group can have a substantial range of potential innervation. Defining the MU count (discussed in detail below in Donor Nerve: Neurophysiological Evaluation) corresponding to that motor score may be critical in surgical decision making. When few axons account for the strength of a potential donor muscle, a tendon transfer is favored. Conversely, when there is a reasonable complement of axons, we favor a nerve transfer.

In the chronic stage following SCI, those myotomes that are primarily paralyzed by an LMN injury eventually become unreceptive to reinnervation..$^{53}$ The muscle groups primarily paralyzed by a UMN injury, though, may retain this capacity according to how well they have maintained LMN innervation. Myotomes immediately adjacent to the site of trauma are often far short of the full complement of axons. Thus, recipient muscle denervation, even in a primarily UMN-affected muscle group, is a potential concern, and as noted above, these muscles will also have undergone collateral reinnervation. But because such reinnervation is not limitless, large numbers of muscle fibers without innervation may remain. After some time without innervation, perhaps $12-18$ months, ${ }^{43}$ these muscle fibers will similarly lose their potential for reinnervation, ${ }^{11}$ and potential recovery of function is greatly reduced.

\section{Clinical Assessment}

From the perspective of reconstructive neurosurgery, 2 questions form the foundation for clinical assessment: 1) What function can be restored? 2) Of the functions that can be restored, which will afford the greatest gain in quality of life given the physical, psychological, and social context of this patient? Once we have identified each patient's favored hierarchy of potential functions to target for restoration, the full arsenal of interventional and noninterventional therapeutic options is reviewed. This includes all therapies within the fields of restorative neurology, reconstructive neurosurgery, and physical medicine and rehabilitation. However, for the typical category of patients with midcervical SCI, the decision is much simpler-elbow extension and grasp and release are primary goals. When nerve transfer surgery is being considered, clinical assessment then proceeds with the aim of thoroughly evaluating potential donor and recipient nerves through a combination of physical examination, neuroimaging, and neurophysiological studies.

In light of occasional unexpectedly poor outcomes in this field, as well as limitations of the clinical examination, our program turned to detailed neurophysiological assessment. Although developed decades ago, ${ }^{18,25,33,49,58}$ quantitative techniques have rarely been used in clinical neurophysiology to date. There is little indication in a traditional clinical neurophysiology practice, but several of the methods have been liberally applied in research over the years, mainly to observe changes over time. ${ }^{26}$ Today, we have a strong clinical need in the form of reconstructive neurosurgery that demands a high degree of 
electromyographic quantification. A critical predictor of the outcome of transferring a nerve is the functioning motor axonal complement within the donor nerve..$^{45}$ To date, neurophysiologists have tended to rely on subjective quantification of the electromyographic interference pattern (IP). However, quantitative neurophysiology has found a clinical home in this context to accompany its established research utility. In an ideal world, we would accurately quantify and characterize the number of functioning MUs in each nerve: donor, recipient, and backup agonist. However, the question in the surgical context (How many motor axons are in a specific nerve at this time?) is very different from that asked in the usual research trial (Over an extended period of time, how much motor axon loss has occurred?). Estimating the number and character of MUs in the various potential donor and recipient combinations is not readily achieved given the technically difficult targets, namely deep muscles, small muscles, muscles surrounded by multiple agonists, acutely or chronically severely denervated muscles, multiple muscles supplied by a single donor nerve, and muscles affected by both a UMN and an LMN injury.

Therefore, our program attempts to refocus established quantitative techniques, as described below, to the clinical arena, adjusted to address the difficulties associated with providing accurate and reliable information relevant to the procedures being considered. We discuss the evaluation of first donor and then recipient nerves, noting initially the pitfalls of the physical examination, followed by the pitfalls of standard neurophysiology, and finally consider how these might be addressed through our current neurophysiological approach.

\section{Donor Nerve: Physical Examination}

Physical evaluation of donor muscles is based on an assessment of bulk, strength, and control. Donor muscles are chosen with the recipient in mind, matched according to known favorable pairings based on function..$^{10,37}$ The concept of a backup agonist to prevent new functional deficit when the donor is eliminated or degraded (for example, infraspinatus for teres minor muscle, biceps for supinator muscle) is important. ${ }^{59}$ Because the region of injury frequently extends both above and below the site of obvious SCI, voluntary activation of the donor muscle can reflect a combination of LMN deficit, sensory deficit (for example, proprioception), and UMN deficit. ${ }^{7,8}$ Several other factors confound the physical exam. The function of a potential donor muscle can be limited by soft tissue changes or spastic co-contraction of the antagonist, masking true functionality. ${ }^{35}$ Deciphering the force generated by a potential donor muscle can be obscured by concurrent agonist muscle activation across the same joint. Muscle bulk can be hard to determine, especially in deep muscles, unless imaging has been undertaken, and the collateral reinnervation discussed above reduces confidence in this parameter as an indicator of innervation. This is most pertinent when assessing a muscle graded as MRC Grade 4 because the underlying innervation can be very poor despite apparent strength and bulk, which would provide an inadequate axon supply for a nerve transfer with a consequently poor outcome.

\section{Donor Nerve: Neurophysiological Evaluation}

In both acute and chronic cervical SCI patients, we predominantly rely on needle electromyography (EMG) during voluntary activation, usually at maximum, to assess the donor nerve. This method is essentially identical to the neurophysiological approach applied to nerve transfer for the typical brachial plexus and peripheral nerve injury. It is uncommon for donor muscles to be absolutely normal, similar to typical brachial plexus injury cases, emphasizing the importance of a detailed neurophysiological study. Many suffer a degree of LMN injury, innervated by segments just above the spinal cord lesion that have been partially affected by the central gray matter injury or concomitant peripheral nerve injury? This manifests electromyographically as chronic neurogenic changes, with large and fast-firing MUPs, and a reduction in the IP. Classic EMG assessment is subjective, usually dividing the IP into normal, reduced, and discrete, with some further characterization based on the number, size, and frequency characteristics of MUPs (Fig. 1). This grading scale does not allow for selection among severely denervated donor nerves, as is often the case. To address this, we use several methods to quantify and decide among potential donors, most frequently MU counting given its ease of application and correlation with outcome (Mandeville et al., unpublished data, 2017). This technique entails identifying and counting individual MUs within the IP at full force in several areas of the donor muscle. Sampling is spread throughout the axis of the muscle and can be guided by ultrasound in deep muscles to ensure adequate coverage of each quadrant. The number of samples depends on muscle size, but often approximately 6 samples are taken. Once the number of units reaches 6 or 7 within an IP, it is usually not possible to count individual MUs unless decomposition software is used (DQEMG ${ }^{51}$ EMGLab, ${ }^{34}$ multi-MUP ${ }^{20}$ ). The technique of such visual MU counting can be optimized in several ways: 1) objective-interactive EMG with manipulation of the needle tip to alter individual MU conformations, 2) alteration of filter settings, and 3 ) the use of commonly preloaded quantification software that can identify and display individual MUs rapidly and usually effectively (multi-MUP ${ }^{20}$ ).

In severely affected donor nerves, categorization is subsequently based on MU counts, from 0 to 6 MUs. Otherwise, when there are 7 or more MUs, the IP is categorized in the usual manner (normal, mild, or moderate severity). The grading system applied to donor nerves at our center is as follows: "excellent" donor, a normal or only mildly affected IP; "good" donor, moderate severity IP; "fair" donor, 5-6 MUs; "questionable" donor, 3-4 MUs; and "poor" donor, 1-2 MUs. Questionable donors might be used when no alternative is available and a solid backup agonist muscle allows transfer of its entire innervation.

When MU count is possibly confounded by a component of UMN dysfunction or when 2 similar donors are being compared, quantification can be further refined: innervation quotient (IQ; Sheean et al., unpublished data, 2017) and turns per second. The IQ involves IP decomposition of individual MUPs, measuring size index ${ }^{41,47}$ (SI) and firing rate (FR). The IQ of a muscle is the average of the individual MUP's SI $\times$ FR product; the greater the 


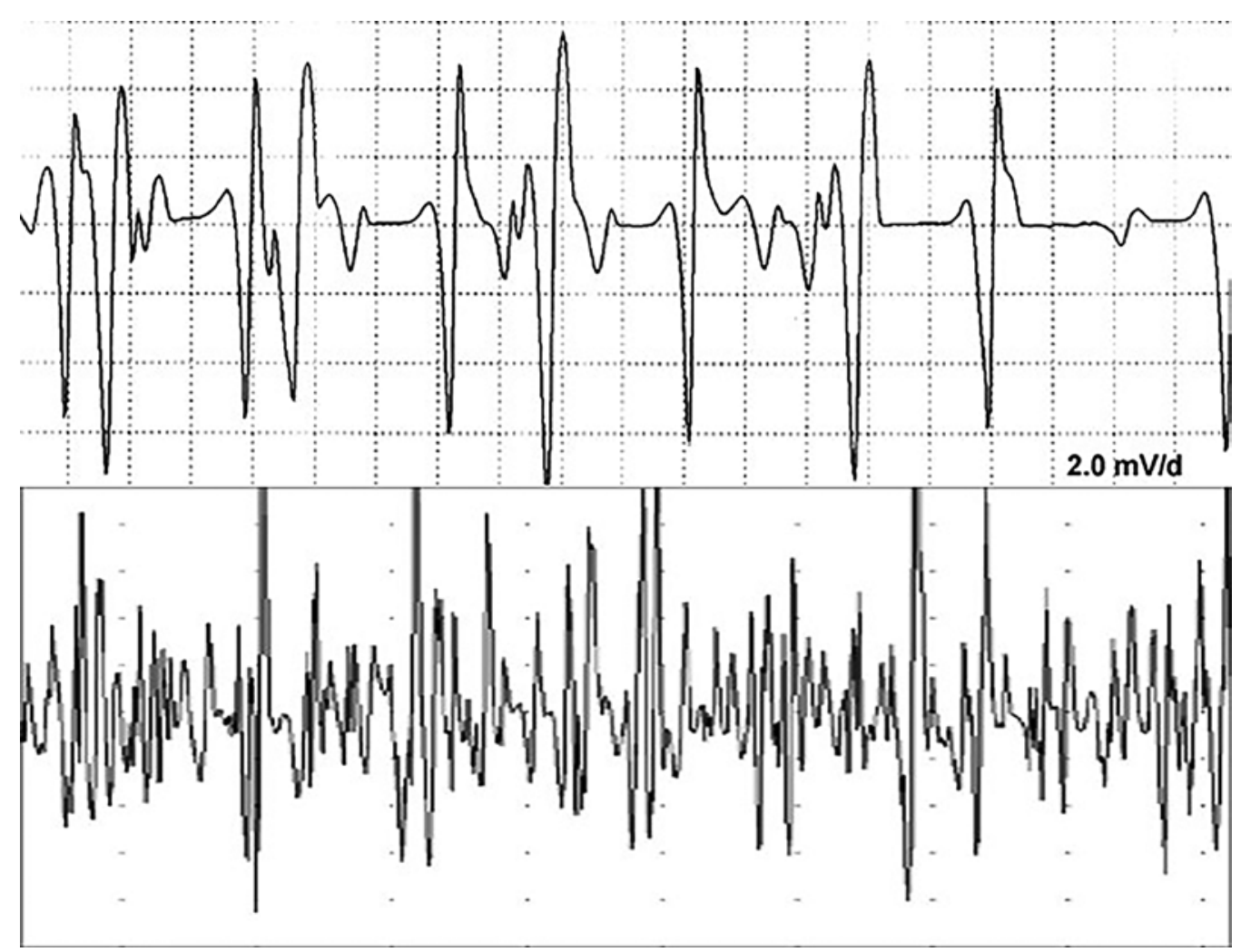

FIG. 1. Upper: Trace showing a discrete IP, a "poor" donor: few MUs (only 2) that are large and discrete. Lower: Trace showing a normal, or near-normal, IP, an "excellent" donor: numerous different MUs obscuring the baseline, too many to count, without significantly increased amplitude.

combined UMN and LMN denervation, the higher the IQ. Turns-amplitude analysis is an established quantitative technique that contains information on the turns (changes in signal polarity) per second in an IP, a parameter validated in predicting recovery after laryngeal fold paralysis. ${ }^{39,52}$ Unstable MUs invalidate the results, but this is usually not an issue in chronic SCI patients. At our center, we have observed a correlation between MU count and turns per second, equating to approximately $1 \mathrm{MU}$ count per 100 turns/sec up to 500 turns/sec (Mandeville et al., unpublished data, 2017). These techniques are sensitive to UMN injury and can be used in conjunction with MU count. They can also distinguish between donor nerves of moderate and mild denervation when visual MU counting is not possible. However, quantification of UMN dysfunction requires further development; techniques such as brain motor control assessment ${ }^{46}$ (BMCA) may provide more information in this regard.

Donor nerve evaluation is also sometimes amenable to MU number estimation ${ }^{2,4,6,26,33}$ (MUNE) techniques (Fig. 2 ). These techniques were specifically developed to directly estimate the number of motor axons supplying an entire muscle-a key goal of neurophysiology in nerve transfer surgery. (This contrasts with the methods described thus far that are surrogates for this estimate and subject to the sampling error and inaccuracy that comes with such an abstraction.) Several variants of MUNE exist, but most are based on obtaining an estimate of the average individual surface-recorded MU amplitude and dividing this into the compound muscle action potential (CMAP) obtained by supramaximal nerve stimulation to approximate the total number of MUs innervating the recipient muscle. Other methods scan the CMAP from subthreshold to supramaximal stimulation ${ }^{2}$ or employ a mathematical model based on IP samples and the CMAP, such as MU number index ${ }^{40}$ (MUNIX). The MUNE techniques have been limited to isolated surface muscles as neighboring co-innervated muscles invalidate surface recordings (for example, extensor carpi radialis longus and brevis muscles). This limitation can be overcome to some degree. Isolating the target muscle can be achieved by recording with the needle electrode shaft (macro-EMG, ${ }^{50}$ Conmac, ${ }^{28}$ or concentric cannula) rather than the surface electrode. At our center, we have used a macro-EMG needle (macro-MUNE), described and validated by de Koning et al. in $1988^{18}$ using the spike-triggered average ${ }^{3,5}$ (STA) MUNE approach. If the muscle is large, then we sample at intervals of $0.5 \mathrm{~cm}$ perpendicular to the motor point. There are limitations to this technique-a muscle size width greater than $1.5 \mathrm{~cm}$ is preferable, near-nerve needle stimulation may be necessary, and time and preparation are required-all reducing its clinical use to only occasional patients. Although requiring further validation, macro-MUNE contributes to enhancing preoperative estimates of functioning donor motor axons. However, no form of MUNE assesses the degree of voluntary control of the MUs counted.

Concurrent use of ultrasound also benefits evaluation of the donor nerve and muscle. Here, its value comes from 

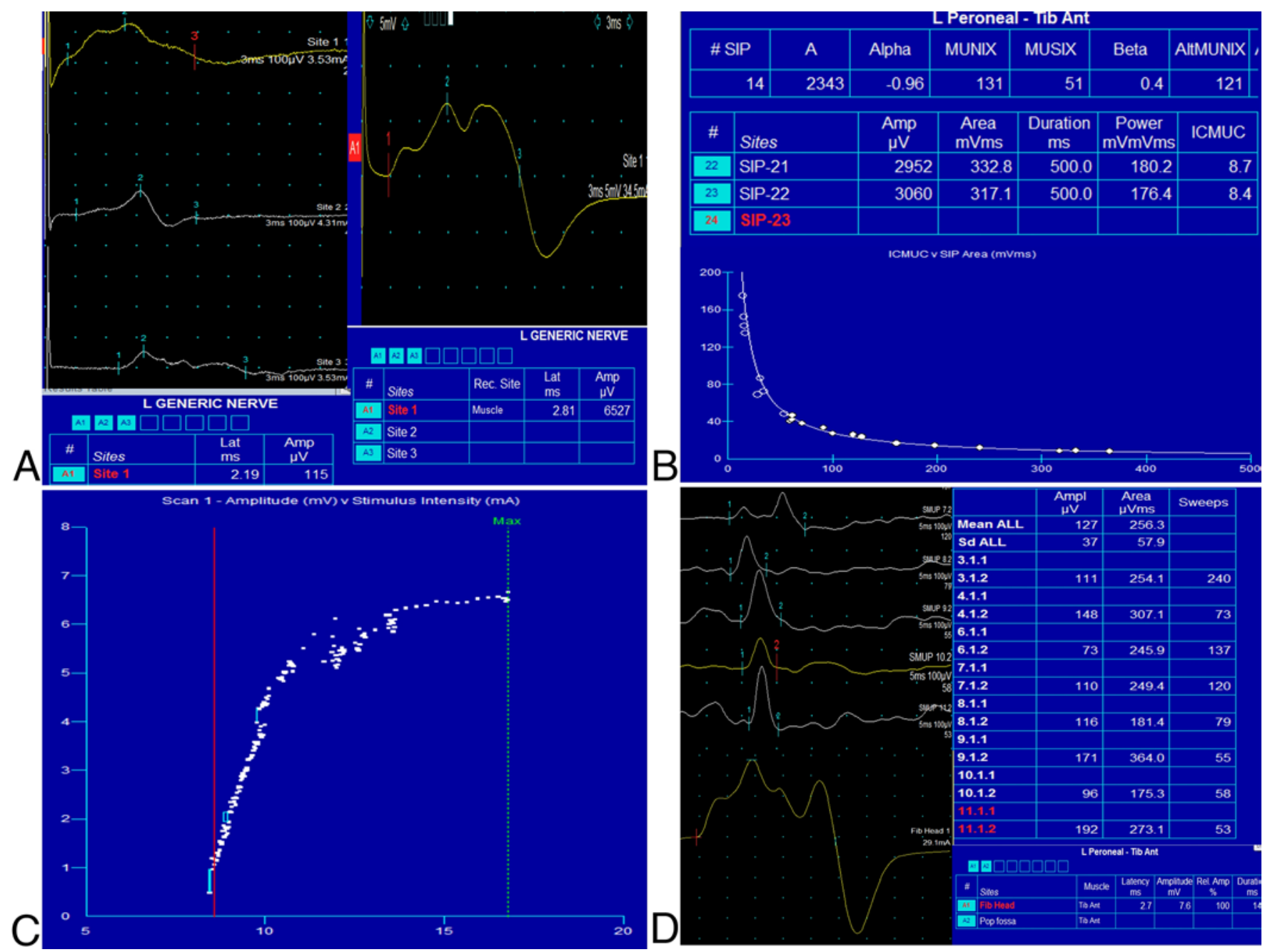

FIG. 2. Four methods of MUNE applied to the same muscle, the tibialis anterior muscle, in a healthy volunteer: multipoint stimulation MUNE that ended up estimating $161 \mathrm{MUs}(\mathbf{A})$; MUNIX, with a value of 131 (this is not a count, B); CMAP scan, a qualitative analysis that returns "step" number and size (C); macro MUNE using the spike-trigger averaging technique that ended up estimating 216 MUs (demonstrating some variability between methods, D).

targeting atrophied or deep muscles for needle EMG recording, echogenic and area characterization of both nerve and muscle, and observation of a muscle's contractility.

In summary, our current approach in determining the propriety of a donor nerve as a source of functioning axons in a nerve transfer surgery heavily relies on the MU count method. Further decomposition of the IP is performed only when the IP is at least moderately reduced and, even then, only in certain circumstances: to investigate a UMN component affecting the MU firing rate, to more accurately compare 2 similar potential donor options, and, lastly, to support the MU count in borderline or hard-to-count cases. In an ideal world, we would obtain an MUNE on every donor nerve-innervated muscle, at least where the IP pattern is moderately affected or worse. For the reasons mentioned above, there are significant limitations that restrict the use of MUNE to only occasional instances. Nevertheless, a prudent approach when evaluating a donor nerve might first consider if MUNE can be used, before moving on to an MU count, with or without further decomposition. Some points are worth noting: MUNE can- not describe any UMN contribution, further research is still required to identify an MUNE threshold that might disqualify a nerve from being a donor, and the value of obtaining an MUNE over that of obtaining an MU count is currently unknown.

\section{Recipient Nerve: Physical Examination}

Recipient muscles, by definition, are very weak or totally paralyzed from UMN and/or LMN deficits. As a rule, a target is not selected for nerve transfer unless its strength is MRC Grade 2 or less and has shown no improvement in strength in recent months. Assessing strength can be difficult. One must consider whether the lack of apparent function is due to a passive range of motion deficit caused by soft tissue changes or simply an inability to overcome spastic antagonist activity (spastic co-contraction). Once strength is determined to be truly less than MRC Grade 3 , we are interested in the degree of LMN deficit in the recipient nerve since a chronically denervated muscle loses its capacity to receive axons. ${ }^{53}$ The degree of LMN injury 


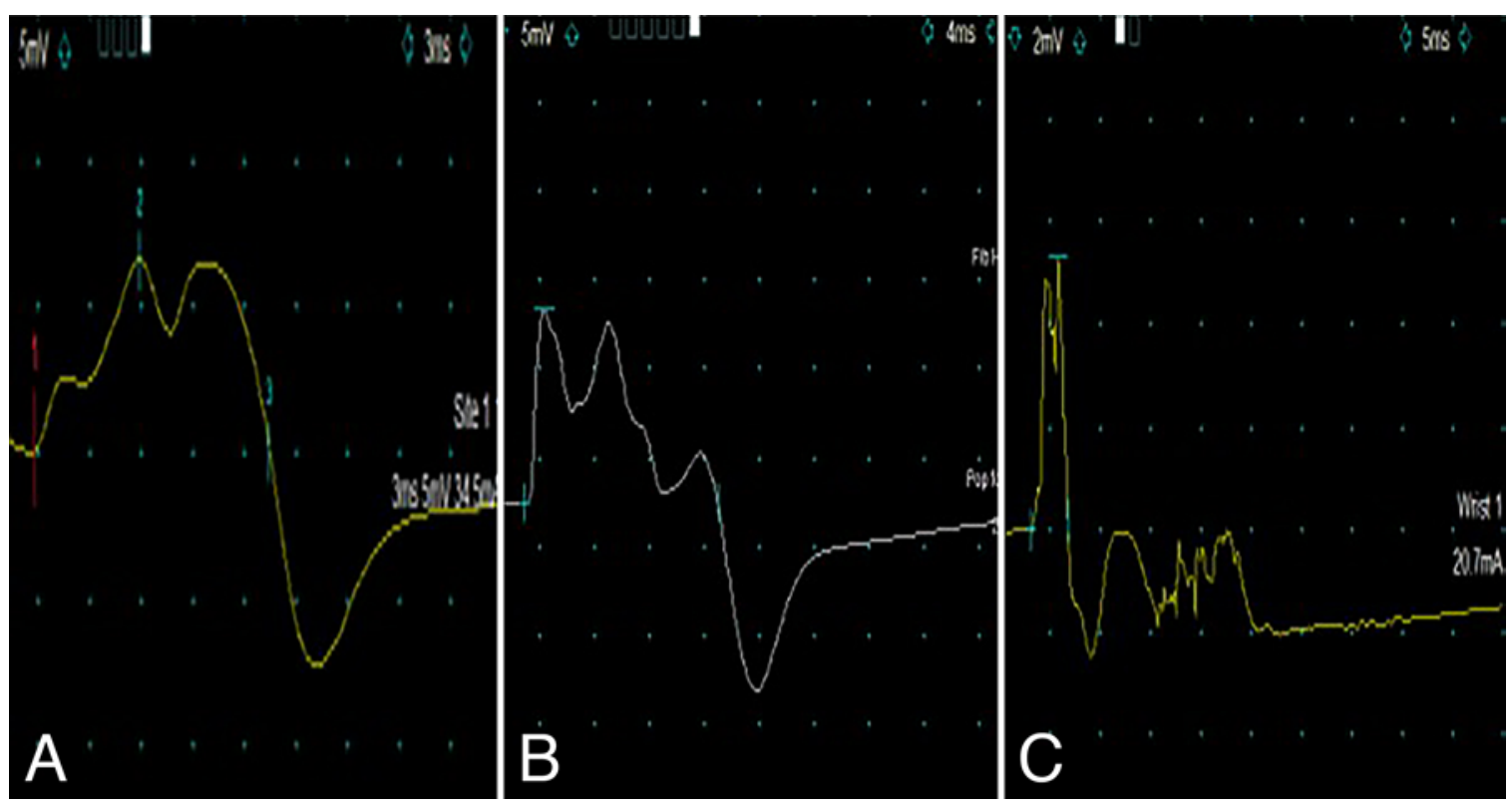

FIG. 3. Variation in CMAP morphology depending on the electrode used in the tibialis anterior muscle of a healthy volunteer: standard surface electrode (A), intramuscular concentric needle electrode shaft (B), and intramuscular concentric needle electrode tip (C). Note the increased sharp components in the latter trace.

can be assessed somewhat by the extent of muscle atrophy, but in some cases this is a poor proxy because bulk alone can be misleading as a result of collateral reinnervation. Another approach to gauging the amount of LMN injury involves eliciting reflexes that activate the target muscle, including deep tendon jerks and Hoffmann's reflex. Nevertheless, reliance on reflex movements alone has limitations. For example, such reflexes are more dominant in the flexor muscles of the upper limb and can be affected by sensory deficits that disrupt the reflex arc., ${ }^{9,38}$ Individual muscle responses can be hard to identify, and not all muscles can be evaluated in this way. Furthermore, the force of reflex movement can be a poor proxy for the degree of LMN injury if collateral reinnervation has taken place. And in any case, the force of the reflex response may be well below the full force that the muscle is capable of.

\section{Recipient Nerve: Neurophysiological Evaluation}

Fundamentally, the goal of recipient muscle evaluation is to quantify the number and character of MUs in the recipient nerve. Again, this becomes relevant only in the chronic period after SCI. Within the 1st year, the quality of innervation of the recipient nerve is essentially irrelevant because the motor endplate remains available. Other than assessing subclinical recovery, no further neurophysiological assessment is required until after this period has passed. Thereafter, we want to ensure that there are axons remaining to preserve motor endplates, even if dysfunctional. We start with supramaximal stimulation of the recipient nerve, which allows for the observation of movement as well as the recording of CMAPs. Subsequently, the use of tetanic stimulation (if tolerated) can allow MRC grading of a muscle's strength or force-generating capacity.

While this approach can be very effective in assessing some muscle groups, there are a number of possible confounders. Restriction of recipient muscle movement by spastic antagonists or soft tissue changes prevents movement or force assessment. For this, we are forced to rely on neurophysiological findings to inform us about recipient nerve innervation. Another possible problem is coactivation of agonists by the nerve stimulus, obscuring the force produced by the recipient muscle. For the problem of agonist coactivation, we can occasionally overcome it by using selective distal branch stimulation as close to the target muscle as possible, by means of a monopolar needle, often with ultrasound guidance. For example, to more accurately assess innervation of the flexor pollicis longus muscle, we specifically stimulate the anterior interosseous nerve rather than the median nerve at the elbow.

The coactivation of neighboring agonists that confound force assessment also affects recording of CMAPs. The use of selective distal branch stimulation can again be of benefit in this situation, allowing more isolated recording of target muscle CMAPs. However, because stimulation of isolated nerve branches is not always possible and target muscles are often unsuitable for surface electrode recording (for example, flexor digitorum profundus muscle), we occasionally employ needle electrode recording. For this recording, more information can be gained when the needle shaft is used to allow recording from an increased surface area, representing a larger area of muscle. Otherwise, the concentric needle tip electrode is used, although not ideally. If a concentric needle electrode is used, the needle is placed well within the belly of the muscle, recording a rapid-rising motor potential with sharp serrated components (Fig. 3C). It is critical to record these measurements with the needle in the muscle of interest. If the needle is outside the muscle, the motor potential is smoother, more like a surface CMAP and more susceptible to contamination by nearby muscle artifact. The goal is to have as much 
of the recording surface within the muscle of interest as possible. The resulting needle shaft-recorded CMAP approximates the regular surface-recorded CMAP but varies somewhat in amplitude and/or area depending on location within the muscle and may have more turns and sharp components (Fig. 3B). Normal values do not exist for this technique, but significant abnormality is suggested by small, difficult to obtain responses with abnormal morphology. Additional information can be gathered when using the concentric needle electrode; an MU count can be performed in severely denervated muscle by applying incremental stimulation. This is the counterpart to the MU count of the volitional IP in the earlier discussion of donor evaluation.

Finally, collateral reinnervation distorts the relationship between innervation and CMAP or force. Collateral reinnervation can result in a small number of very large residual MUPs, and the muscle bulk, stimulated force, and CMAP can be deceptively good. Even in an ideal scenario in which donor axons significantly outnumber recipient axons, these donor axons may not achieve the presurgical level of recipient muscle innervation because an unknown proportion of the denervated recipient tubules will fail to receive a donor axon. ${ }^{11,22,24,53}$ If sufficient in number and territorial distribution, it is possible that those donated axons making it to the target muscle will be able to reinnervate all muscle fibers and thus eventually reproduce the force and CMAP observed preoperatively with stimulation, assuming full UMN control. However, if significant chronic LMN denervation was originally present, the inevitable postsurgical pruning across the coaptation site from misdirection of regenerating axons into inappropriate endoneurial tubes may, among other factors, ${ }^{11}$ result in an insufficient supply (or coverage within the muscle) to reinnervate all previously innervated muscle fibers. This may result in a failure to achieve the force predicted from preoperative stimulation. In this situation, we find the technique of MUNE, as described in Donor Nerve: Neurophysiological Evaluation, to be informative, if feasible.

At our center, we find the use of ultrasound highly beneficial to aid the neurophysiological studies when evaluating recipient muscles and nerves. Ultrasound yields several benefits: 1) targeting of atrophied or deep muscles for needle CMAP recording, 2) guidance of the needle electrode for selective near-nerve stimulation or block, and 3) echogenic and area characterization of both nerve and muscle ${ }^{42,57}$ corroborating imaging characteristics with electrophysiological function. Correct targeting of a muscle is also aided in the forearm (that is, deep and superficial finger flexors) through direct muscle needle stimulation and subsequent observation of movement, as well as passive extension/flexion of individual finger joints, with correct needle placement indicated by maximal needle movement.

In summary, our approach to the assessment of a recipient nerve's suitability to receive axons relies on stimulation of the distal branches supplying the recipient muscle, with subsequent observation of movement and force, as well as recording of CMAPs either by surface or needle electrode. The application of MUNE techniques described above is decided case by case, albeit more often and per- haps more usefully than in donor nerve evaluation. Again, as with donor nerve evaluation, a prudent approach might always consider whether an MUNE can be acquired after obtaining a CMAP and observing movement and force. Nevertheless, further validation is required and is ongoing.

\section{Summary}

We have presented our approach to the most conspicuous drawbacks of physical examination and standard neurophysiological studies when evaluating donor and recipient nerves for nerve transfer in SCI. Donor nerve evaluation can deceive in terms of functional axonal content through apparently adequate bulk, force, and CMAPs. In particular, a donor with seemingly good strength, an MRC Grade 4, but significant collateral reinnervation may make for a poor outcome. In the hope of avoiding such unexpectedly poor surgical outcomes, we have discussed several quantitative approaches of varying complexity that help to address this predicament. Recipient muscle denervated within the last year remains receptive to reinnervation and is simply evaluated for subclinical native innervation at the completion of collateral sprouting. Recipient muscle chronically paralyzed by a UMN injury can lead to similar deception. Functional axonal content can appear good through apparently adequate bulk and a reasonable CMAP. We have presented several quantitative techniques that can be applied in different situations to avoid being misled in this regard.

\section{Conclusions}

Given the pitfalls of the clinical assessment of potential donor and recipient muscles, there is a clear role for neurophysiological assessment. The 2 main problems to avoid are 1) transferring nerves from a muscle that will leave it too weak, if no backup agonist is present, and a recipient muscle with a graft containing too few axons under voluntary control; and 2) transferring nerves to a recipient muscle in the chronic period following SCI that is also too heavily denervated to be able to adequately reinnervate. The current objective and semi-objective evaluation practiced in our program helps to avoid the most glaring examples of the 2 main problems. There are technical issues to overcome with a neurophysiological evaluation, and quantification in this context is in its infancy. However, quantitative work from decades ago has found a new home in the clinical arena and should be explored and validated further. It is hoped that this paper will stimulate interest, discussion, and research to advance preoperative and intraoperative assessment to deliver better surgical outcomes.

\section{References}

1. Bishop B: Neural plasticity: Part 3. Responses to lesions in the peripheral nervous system. Phys Ther 62:1275-1282, 1982

2. Blok JH, Ruitenberg A, Maathuis EM, Visser GH: The electrophysiological muscle scan. Muscle Nerve 36:436-446, 2007

3. Boe SG, Stashuk DW, Doherty TJ: Motor unit number estimation by decomposition-enhanced spike-triggered averaging: control data, test-retest reliability, and contractile level effects. Muscle Nerve 29:693-699, 2004 
4. Bostock H: Estimating motor unit numbers from a CMAP scan. Muscle Nerve 53:889-896, 2016

5. Bromberg MB: Motor unit estimation: reproducibility of the spike-triggered averaging technique in normal and ALS subjects. Muscle Nerve 16:466-471, 1993

6. Bromberg MB: Updating motor unit number estimation (MUNE). Clin Neurophysiol 118:1-8, 2007

7. Brown JM: Nerve transfers in tetraplegia I: Background and technique. Surg Neurol Int 2:121, 2011

8. Brown JM: The reconstructive neurosurgery of spinal cord injury, in Dimitrijevic MR, Kakulas BA, McKay WB, et al (eds): Restorative Neurology of Spinal Cord Injury. New York: Oxford University Press, 2012

9. Brown JM, Kakulas BA: Restorative neurology: past, present, and future. Clin Neurol Neurosurg 114:524-527, 2012

10. Brown JM, Mackinnon SE: Nerve transfers in the forearm and hand. Hand Clin 24:319-340, v, 2008

11. Brown JM, Shah MN, Mackinnon SE: Distal nerve transfers: a biology-based rationale. Neurosurg Focus 26(2):E12, 2009

12. Brown JM, Vivio N, Sheean GL: The clinical practice of reconstructive neurosurgery. Clin Neurol Neurosurg 114:506-514, 2012

13. Brown MC, Ironton R: Sprouting and regression of neuromuscular synapses in partially denervated mammalian muscles. J Physiol 278:325-348, 1978

14. Bryden AM, Hoyen HA, Keith MW, Mejia M, Kilgore KL, Nemunaitis GA: Upper extremity assessment in tetraplegia: the importance of differentiating between upper and lower motor neuron paralysis. Arch Phys Med Rehabil 97 (6 Suppl):S97-S104, 2016

15. Burt AA: The epidemiology, natural history and prognosis of spinal cord injury. Curr Orthop 18:26-32, 2004

16. Cain SA, Gohritz A, Fridén J, van Zyl N: Review of upper extremity nerve transfer in cervical spinal cord injury. J Brachial Plex Peripher Nerve Inj 10:e34-e42, 2015

17. Cashman NR, Maselli R, Wollmann RL, Roos R, Simon R, Antel JP: Late denervation in patients with antecedent paralytic poliomyelitis. N Engl J Med 317:7-12, 1987

18. de Koning P, Wieneke GH, van der Most van Spijk D, van Huffelen AC, Gispen WH, Jennekens FG: Estimation of the number of motor units based on macro-EMG. J Neurol Neurosurg Psychiatry 51:403-411, 1988

19. Erminio F, Buchthal F, Rosenfalck P: Motor unit territory and muscle fiber concentration in paresis due to peripheral nerve injury and anterior horn cell involvement. Neurology 9:657-671, 1959

20. Falck B, Stålberg E, Stålberg S, Aström M: Multi-MUP EMG analysis in clinical routine. Neurol Neurochir Pol 30 (Suppl 3):55-70, 1996

21. Feinberg J: EMG: myths and facts. HSS J 2:19-21, 2006

22. Fenrich K, Gordon T: Canadian Association of Neuroscience review: axonal regeneration in the peripheral and central nervous systems - current issues and advances. Can J Neurol Sci 31:142-156, 2004

23. Fridén J, Gohritz A: Brachialis-to-extensor carpi radialis longus selective nerve transfer to restore wrist extension in tetraplegia: case report. J Hand Surg Am 37:1606-1608, 2012

24. Fu SY, Gordon T: Contributing factors to poor functional recovery after delayed nerve repair: prolonged denervation. J Neurosci 15:3886-3895, 1995

25. Fuglsang-Frederiksen A, Lo Monaco M, Dahl K: Turns analysis (peak ratio) in EMG using the mean amplitude as a substitute of force measurement. Electroencephalogr Clin Neurophysiol 60:225-227, 1985

26. Gooch CL, Doherty TJ, Chan KM, Bromberg MB, Lewis RA, Stashuk DW, et al: Motor unit number estimation: a technology and literature review. Muscle Nerve 50:884-893, 2014
27. Gordon T, Yang JF, Ayer K, Stein RB, Tyreman N: Recovery potential of muscle after partial denervation: a comparison between rats and humans. Brain Res Bull 30:477-482, 1993

28. Gorio A, Marini P, Zanoni R: Muscle reinnervation-III. Motoneuron sprouting capacity, enhancement by exogenous gangliosides. Neuroscience 8:417-429, 1983

29. Jabre JF: Concentric macro electromyography. Muscle Nerve 14:820-825, 1991

30. Jacob JM, Robbins N: Age differences in morphology of reinnervation of partially denervated mouse muscle. J Neurosci 10:1530-1540, 1990

31. Kugelberg E, Edström L, Abbruzzese M: Mapping of motor units in experimentally reinnervated rat muscle. Interpretation of histochemical and atrophic fibre patterns in neurogenic lesions. J Neurol Neurosurg Psychiatry 33:319-329, 1970

32. Maynard FM Jr, Bracken MB, Creasey G, Ditunno JF Jr, Donovan WH, Ducker TB, et al: International standards for neurological and functional classification of spinal cord injury. Spinal Cord 35:266-274, 1997

33. McComas AJ, Fawcett PRW, Campbell MJ, Sica REP: Electrophysiological estimation of the number of motor units within a human muscle. J Neurol Neurosurg Psychiatry 34:121-131, 1971

34. McGill KC, Lateva ZC, Marateb HR: EMGLAB: an interactive EMG decomposition program. J Neurosci Methods 149:121-133, 2005

35. McKay WB, Arthur Sherwood AS, Simon Tang ST: Neurophysiological assessment of human motor control and changes caused by spinal cord injury, in Dimitrijevic MR, Kakulas BA, McKay WB, et al (eds): Restorative Neurology of Spinal Cord Injury. New York: Oxford University Press, 2012

36. Merrell GA, Barrie KA, Katz DL, Wolfe SW: Results of nerve transfer techniques for restoration of shoulder and elbow function in the context of a meta-analysis of the English literature. J Hand Surg Am 26:303-314, 2001

37. Midha R: Nerve transfers for severe brachial plexus injuries: a review. Neurosurg Focus 16(5):E5, 2004

38. Minassian K, Hofstoetter U, Tansey K, Mayr W: Neuromodulation of lower limb motor control in restorative neurology. Clin Neurol Neurosurg 114:489-497, 2012

39. Munin MC, Rosen CA, Zullo T: Utility of laryngeal electromyography in predicting recovery after vocal fold paralysis. Arch Phys Med Rehabil 84:1150-1153, 2003

40. Nandedkar SD, Nandedkar DS, Barkhaus PE, Stalberg EV: Motor unit number index (MUNIX). IEEE Trans Biomed Eng 51:2209-2211, 2004

41. Okajima Y, Tomita Y, Sasa H, Tanaka N, Kimura A, Chino $\mathrm{N}$ : The size index as a motor unit identifier in electromyography examined by numerical calculation. J Electromyogr Kinesiol 9:201-208, 1999

42. Pillen S, van Alfen N: Skeletal muscle ultrasound. Neurol Res 33:1016-1024, 2011

43. Pindrik J, Chhabra A, Belzberg AJ: Update on peripheral nerve surgery. Neurosurgery 60 (Suppl 1):70-77, 2013

44. Rafuse VF, Gordon T, Orozco R: Proportional enlargement of motor units after partial denervation of cat triceps surae muscles. J Neurophysiol 68:1261-1276, 1992

45. Schreiber JJ, Byun DJ, Khair MM, Rosenblatt L, Lee SK, Wolfe SW: Optimal axon counts for brachial plexus nerve transfers to restore elbow flexion. Plast Reconstr Surg 135:135e-141e, 2015

46. Sherwood AM, Dimitrijevic MR: Comprehensive assessment of control of motor activity: brain motor control assessment (BMCA). Phys Med Biol 33 Suppl:163, 1988

47. Sonoo M, Stålberg E: The ability of MUP parameters to discriminate between normal and neurogenic MUPs in concentric EMG: analysis of the MUP "thickness" and the proposal 
of "size index". Electroencephalogr Clin Neurophysiol 89:291-303, 1993

48. Stålberg E: Macro electromyography, an update. Muscle Nerve 44:292-302, 2011

49. Stålberg E: Macro EMG, a new recording technique. J Neurol Neurosurg Psychiatry 43:475-482, 1980

50. Stålberg E, Grimby G: Dynamic electromyography and muscle biopsy changes in a 4-year follow-up: study of patients with a history of polio. Muscle Nerve 18:699-707, 1995

51. Stashuk DW: Decomposition and quantitative analysis of clinical electromyographic signals. Med Eng Phys 21:389404, 1999

52. Statham MM, Rosen CA, Nandedkar SD, Munin MC: Quantitative laryngeal electromyography: turns and amplitude analysis. Laryngoscope 120:2036-2041, 2010

53. Sulaiman W, Gordon T: Neurobiology of peripheral nerve injury, regeneration, and functional recovery: from bench top research to bedside application. Ochsner J 13:100-108, 2013

54. Thompson W, Jansen JK: The extent of sprouting of remaining motor units in partly denervated immature and adult rat soleus muscle. Neuroscience 2:523-535, 1977

55. van Zyl N, Hahn JB, Cooper CA, Weymouth MD, Flood SJ, Galea MP: Upper limb reinnervation in C6 tetraplegia using a triple nerve transfer: case report. J Hand Surg Am 39:1779-1783, 2014

56. Wade DT, Hewer RL: Functional abilities after stroke: measurement, natural history and prognosis. J Neurol Neurosurg Psychiatry 50:177-182, 1987

57. Walker FO, Cartwright MS, Wiesler ER, Caress J: Ultrasound of nerve and muscle. Clin Neurophysiol 115:495-507, 2004
58. Walton JN: The electromyogram in myopathy: analysis with the audio-frequency spectrometer. J Neurol Neurosurg Psychiatry 15:219-226, 1952

59. Weber RV, MacKinnon SE: Nerve transfers in the upper extremity. J Am Soc Surg Hand 4:200-213, 2004

60. Wohlfart G: Collateral regeneration in partially denervated muscles. Neurology 8:175-180, 1958

\section{Disclosures}

The authors report no conflict of interest concerning the materials or methods used in this study or the findings specified in this paper.

\section{Author Contributions}

Conception and design: all authors. Drafting the article: all authors. Critically revising the article: all authors. Reviewed submitted version of manuscript: Mandeville. Approved the final version of the manuscript on behalf of all authors: Mandeville. Administrative/technical/material support: Mandeville.

\section{Supplemental Information \\ Videos \\ Video Abstract. https://vimeo.com/220787946.}

\section{Correspondence}

Ross Mandeville, Department of Neurology, UCSD Medical Center, 402 Dickinson St., Ste. 1-190, San Diego, CA 92103-8465. email: rmandeville@ucsd.edu. 